\title{
An adaptive weight adjustment algorithm based on optimization of weight distance similarity
}

\author{
Ningguo Yang, Xintao Ren, Jianwei Zhang, Weibing Jiang \\ Baicheng Ordnance Test Center of China, Baicheng 137001, Jilin, China \\ Email: yng7587@163.com
}

\begin{abstract}
Keywords: AHP, entropy weight method, distance similarity, adaptive weight adjustment. Abstract: In other to enhance the assessment ability to modernized weapons, an adaptive weight adjustment algorithm based optimization of weighted-distance similarity is proposed. Adopting Analytic Hierarchy Process and entropy weight method to evaluate the subjective and objective weights of indices, building the model of comprehensive weight computing based optimization of weight distance similarity, designing the adaptive adjustment method with comprehensive weight, the accuracy and maneuverability are proved by giving example analysis and the result shows that the algorithm can be used as a reference in the weapon evaluation system construction.
\end{abstract}

\section{Introduction}

With rapid development of military equipment type test model and rapid transformation of combat system, weapons system competition and choosing excellent become the necessery mode in the process of equipment development, therefore, performance evaluation and decision turn into the most important thing. The key of acquiring a scientific assessment result is how to evaluate weight reasonably, hence ascertaining the weight scientifically would be the significant role in the appraisal system from now on.

Nowadays the methods to confirm the weight include subjective method, objective method and subjective and objective synthetic method. The subjective method including expert scoring, delphi method, AHP and so on, whose advantage are according to practical problems the experts not only analyze the order of index weight but also could not lead to concluded index weight going against the importance degree of actural problems ${ }^{[1]}$, whose disadvantage are the objective factors affects results substantially, the results are rough, it is not suitable for the problem with higher accuracy requirement. The objective method including Entropy Method, PCA and so on, whose advantage is owing to adopting objective calculation, avoid the effect of human, however, the degree of participation of weight is low, and the case may happen that assessing members have different views in the cognitive degree of weights ${ }^{[2]}$. In resent years scholars have processed many subjective and objective comprehensive assignment weight determination methods, which make up for the weaknesses of subjective and objective method, and which reache better results. But those researches often regard the assessing members as the passive to synthesize objectivly in the study of synthesizing integrated weight $^{[3-7]}$.

On the basis of previous studies, an adaptive weight adjustment algorithm based optimization of weight distance similarity method is proposed in this paper, in practice of weapon system of assessing index weight solution. Firstly, the method using AHP and Entropy Method to determine the subjective and objective weight of the assessing index and secondly, according to weight distance similarity of every inedx weight the subjective and objective weight and lastly by iterative and adaptive adjustment conclude the comprehensive weight of indices.

\section{Adaptive Weight Adjustment Algorithm}

Assume in the evaluation system, there are $\mathrm{m}$ schemes to be assessed, $\mathrm{n}$ assessing index, $\mathrm{k}$ experts that participate in the assessment. Then evaluation schemes and indexes conpose an evaluation matrix $\left(x_{i j}\right)_{m \times n}$, through the standard handlng of evaluation matrix, indexes would unite into the benefit index to get the normalized matrix $\left(x_{i j}^{\prime}\right)_{m \times n}$ 


\section{Index Weigh Determination.}

When calculate the index weight, firstly the assessing indexes are compared by k experts, adopting AHP(a subjective method) the weight of every assessing indexes $W=\left\{W_{1}, W_{2}, \mathrm{~L} W_{m}\right\}$ are determined to satisfy the conditions of $0 \leq W_{i} \leq 1$ and $\sum_{i=1}^{n} W_{i}=1$. Secondly, according to extent of variation of every indexes, Entropy Method is used to calculate every index weight so that the weight $W^{\prime}=\left\{W_{1}^{\prime}, W_{2}^{\prime}, \mathrm{L} W_{m}^{\prime}\right\}$ that sastify the conditions of $0 \leq W_{i} \leq 1$ and $\sum_{i=1}^{n} W_{i}=1$ is obtained. When generating comprehensive assessing weight of the index, in the first place we make definition as follows:

The distance similarity degree between corresponding different weights of the same indexes, which is devoted by $\mathrm{r}$ :

$$
r_{i}=1-\frac{\left|W_{i}-W_{i}^{\prime}\right|}{\sum_{i=1}^{n}\left|W_{i}-W_{i}^{\prime}\right|}
$$

where, $0 \leq r_{i} \leq 1$ and $\sum_{i=1}^{n} r_{i}=1,0 \leq w_{i} \leq 1$ and $\sum_{i=1}^{n} w_{i}=1$.

The larger the value of $r_{i}$ is, showing that on the condition of this index, the shorter the distance between weights obtained by subjective method and weights obtained by objective method is, the higher the similarity is, so objective weight should play a leading role, when detering the weight, to optimize the subjective weights and make the comprehensive weight applicable for the assessing problem with higher accuracy requirement. The smaller the value of $r_{i}$ is, showing that on the condition of this index, the lower the similarity between subjective and objective weight is, and hence subjective weight should play a leading role in the process of weight combination in order to avoid the problem of inverted sequence caused by objective weights.

Based on the above analysis, we conclude the comprehensive weight of index $i$ is

$$
w_{i}=\frac{\left(1-r_{i}\right) W_{i}+r_{i} W_{i}^{\prime}}{\sum_{i=1}^{n}\left[\left(1-r_{i}\right) W_{i}+r_{i} W_{i}^{\prime}\right]}
$$

where, $0 \leq w_{i} \leq 1, \quad \sum_{i=1}^{n} w_{i}=1$.

\section{Adaptive Adjustment of Index Weight}

In the comprehensive index weight $\mathrm{w}$ completed by Eq. 2, the importance degree of every index may have conflicts with the importance degree obtained by expert scoring method. In order to solve the problem, comparing the logic important degree of every index in synthetical weight $\mathrm{W}$ with it in subjective weight $\mathrm{w}$ obtained by AHP, if the two degrees are same, we select was final comprehensive index, or we process $\mathrm{W}$ and $\mathrm{W}$ according to Eq. 3and Eq. 4, untill the logic important degrees of them are same.

$$
r_{i}^{k}=1-\frac{\left|W_{i}-w_{i}^{k}\right|}{\sum_{i=1}^{n}\left|W_{i}-w_{i}^{k}\right|}
$$


where, $0 \leq r_{i}^{k} \leq 1, \sum_{i=1}^{n} r_{i}^{k}=1$

$$
w_{i}^{k+1}=\frac{\left(1-r_{i}^{k}\right) W_{i}+r_{i}^{k} w_{i}^{k}}{\sum_{i=1}^{n}\left[\left(1-r_{i}^{k}\right) W_{i}+r_{i}^{k} w_{i}^{k}\right]}
$$

where, $0 \leq w_{i}^{k+1} \leq 1, \sum_{i=1}^{n} w_{i}^{k+1}=1$

In the two relations $r_{i}^{k}$ is weight distance similarity of the index $\mathrm{i}$ at kth iteration and $w_{i}^{k+1}$ is the comprehensive weight at No. $(\mathrm{k}+1)$ iteration

\section{Example Verification and Analysis}

A certain country decide to send out invitations to research institutes, big military companies, and so on, for artillery hunting radar, and there are many units bid for the project, after examining at all levels, competition and preselection, finally 5 companies meet the condition of competitive bidding, and they participate in the competitive military equipment tender test, the main results of which are as shown in Table 1.

Table 1 assessing index and main results of a certain radar bid test

\begin{tabular}{|c|c|c|c|c|}
\hline Bid company & $\mathrm{a}$ & $\mathrm{b}$ & $\mathrm{c}$ & $\mathrm{d}$ \\
\hline $\begin{array}{c}\text { Bid price/ten } \\
\text { Thousand[yuan] }\end{array}$ & 3600 & 4000 & 3200 & 3000 \\
\hline Ability of multiple targets & 12 & 16 & 14 & 13 \\
\hline Detection range[km] & 300 & 360 & 240 & 280 \\
\hline Position accuracy ECP[m] & 200 & 150 & 180 & 120 \\
\hline System response time/s & 10 & 5 & 6 & 8 \\
\hline jamming source orientation accuracy[mil] & 15 & 20 & 25 & 23 \\
\hline $\begin{array}{c}\text { FH points } \\
\text { Switch time of Fighting and } \\
\text { processions[min] }\end{array}$ & 18 & 20 & 10 & 15 \\
\hline Position false alarm rate/hours per time & 10 & 35 & 25 & 20 \\
\hline
\end{tabular}

In Table 1, ability of multiple targets, detection range, $\mathrm{FH}$ points and position false alarm rate indexes belong to benefit index, and other indexes belong to cost index. We can obtain the normalized matix as shown in Eq. 5, by means of standardizing the indexes in Table 1.

$$
X^{\prime}=\left[\begin{array}{lllllllll}
0.4 & 0 & 0.5 & 0 & 0 & 1 & 0.8 & 0.33 & 0 \\
0 & 1 & 1 & 0.63 & 1 & 0.5 & 1 & 0 & 0.67 \\
0.8 & 0.5 & 0 & 0.25 & 0.8 & 0 & 0 & 0.67 & 0.33 \\
1 & 0.25 & 0.33 & 1 & 0.4 & 0.2 & 0.5 & 1 & 1
\end{array}\right]
$$

\section{Calculating Comprehensive Weight of Assessing Index}

AHP method is adopted to calculate the subjective weight, and judgement matrix discuessed by experts is described by Eq. 7 . When $C R=0.0279<0.1$, the calculated weight of every index is(computation process is omitted):

$$
W=\{0.0898,0.1082,0.1146,0.1795,0.1144,0.1073,0.1049,0.0662,0.1189\}
$$




$$
A=\left[\begin{array}{lllllllll}
1 & 1 / 2 & 1 / 2 & 1 / 3 & 1 & 1 & 1 & 2 & 1 \\
2 & 1 & 1 & 1 / 2 & 1 & 1 & 1 & 2 & 1 / 2 \\
2 & 1 & 1 & 1 / 2 & 1 & 1 & 1 & 2 & 1 \\
3 & 2 & 2 & 1 & 1 & 1 & 2 & 3 & 1 \\
1 & 1 & 1 & 1 & 1 & 1 & 1 & 2 & 1 \\
1 & 1 & 1 & 1 & 1 & 1 & 1 & 1 & 1 \\
1 & 1 & 1 & 1 / 2 & 1 & 1 & 1 & 2 & 1 \\
1 / 2 & 1 / 2 & 1 / 2 & 1 / 3 & 1 / 2 & 1 & 1 / 2 & 1 & 1 \\
1 & 2 & 1 & 1 & 1 & 1 & 1 & 1 & 1
\end{array}\right]
$$

Entropy Method is adopted to determine the objective weight ${ }^{[10,11]}$, the weight of every index is(computation process is omitted):

$$
W^{\prime}=\{0.1006,0.1238,0.1129,0.1195,0.1006,0.1329,0.0934,0.1081,0.1081\}
$$

According to Eq. 2, Eq. 3 and (4), after 14 times of iterative adaptive adjustment the coprehensive weight of the index is

$$
w=\{0.0921,0.1107,0.1126,0.1761,0.1114,0.1100,0.1020,0.0691,0.1160\}
$$

Comparing comprehensive weight $w$ with the weight determined by AHP method, we can see that, the logic important degree of every assessing index remain unchanged, namely the obtained comprehensive weight of the index can reflect the experts' subjective attitude to important gegree of actual problems, thus, through the iterative adaptive adjustment, the comprehensive weight can be modified objectively to make it more scientific and reasonable and we could use it in decision evaluation with higher accuracy requirements.

\section{Evaluation and Analysis Based TOPSIS Method}

Through the multiplying normalized matrix $X^{\prime}$ by the matrix calculated by the comprehensive weight $\mathrm{w}$, we get the judgment matrix

$$
Z=\left[\begin{array}{lllllllll}
0.0369 & 0 & 0.0563 & 0 & 0 & 0.11 & 0.0816 & 0.0228 & 0 \\
0 & 0.1107 & 0.1126 & 0.111 & 0.1114 & 0.055 & 0.102 & 0 & 0.0777 \\
0.0737 & 0.0554 & 0 & 0.044 & 0.0891 & 0 & 0 & 0.0463 & 0.0383 \\
0.0921 & 0.0277 & 0.0372 & 0.1761 & 0.0446 & 0.022 & 0.051 & 0.0691 & 0.116
\end{array}\right]
$$

The Euclidean distance between every target value and positive and minus ideal solutions is compluted, and the relative closeness of every target is counted, the results of which are shown in Table 2.

Table 2 relative closeness and ranking result of different bid companies index value and optimum value

\begin{tabular}{|c|c|c|c|c|}
\hline Bid company & $\mathrm{D}+$ & $\mathrm{D}-$ & $\mathrm{Ci}$ & ranking result \\
\hline $\mathrm{a}$ & 0.2791 & 0.1543 & 0.3560 & 4 \\
\hline $\mathrm{b}$ & 0.1729 & 0.2572 & 0.5980 & 2 \\
\hline $\mathrm{c}$ & 0.2512 & 0.1483 & 0.3712 & 3 \\
\hline $\mathrm{d}$ & 0.1655 & 0.2549 & 0.6063 & 1 \\
\hline
\end{tabular}

We can find the results of bid company $\mathrm{d}$ is the best in Table 2.

\section{Conclusion}

Based on the previous research, this article propose a new subjective and objective comprehensive method of index evaluation weight calculation. The method adapts AHP which is one of subjective 
method to determine subjective weights and determine objective weights through entropy weight method. When calculating comprehensive weights, the author fully consideres, on the premise of not violate the experts' opinions to the importance degree of actural promems, use objective weight to optimize the subjective weight according to distance similary, and weight the subjective weight adaptivly.Through evaluation and analysis of real example, we prove the feasibility of the algorithm and the algorithm provide a reference for weapons' assessing and determining index weight

\section{Acknowledgements}

The author wishes to thank all my colleagues who previously provided technical support.

\section{References}

[1] Wenge Wang, Zhenxi Wang. Vol.33 No.8:123-125.

[2] Bin Yan, Jingyu Yan, Chao Guo. Journal of Shenyang Agricultural University, 2014-02,45(1):58-61.

[3] Yi Sun, Haifeng Huang, Jianhua Ding, Computer Engineering and Applations, 2014,50(2):35-38.

[4] Wei Yang, Jiarong Shi, Yongfeng Pang, Fuzzy Systems and Mathematics. Vol.28, No.2, Apr. 2014:144-151.

[5] Qiang Ma, Yajun Guo, Yingmin Guo, Systems Engineering and Electronics. Vol.35, No.5, May 2013:1008-1012.

[6] Huajun Zhang, Hui Luo, Control and Decision. Vol.29, No.8, Aug.2014:1471-1475.

[7] Yanping Li, Qi Qiao, Fahe Chai. Research of Environmental Sciences, Vol.27, No.3, Mar.2014:334-340.

[8] Jin Xue, Weijin Chen, Hong Wang. China medical equipment. Vol.28, No.12:44-47.

[9] Yanxiang Huang, Yongfa Song. Journal of Engineering Management. Vol.28, No.3, Jun.2014:057-061.

[10] Shu-zhen Chu, Hui Tang. Drug evaluation in China. Vol.30, No.5, 2013:295-297. 